\title{
Intragenomic Conflict over Dispersal
}

\author{
Elizabeth J. Farrell, ${ }^{1}$ Francisco Úbeda, ${ }^{2}$ and Andy Gardner ${ }^{3, *}$ \\ 1. Wadham College, Parks Road, Oxford OX1 3PN, United Kingdom; 2. School of Biological Sciences, Royal Holloway University \\ of London, Egham TW20 0EX, United Kingdom; 3. School of Biology, University of St. Andrews, St. Andrews KY16 9TH, \\ United Kingdom
}

Submitted January 28, 2015; Accepted April 1, 2015; Electronically published July 8, 2015

Online enhancement: zip file.

\begin{abstract}
A BSTRACT: Intragenomic conflict may arise when social partners are more related through one parent than the other-for example, owing to individuals or gametes of one sex dispersing further prior to fertilization. In particular, genes originating from the former parent are favored to promote selflessness, and those originating from the latter parent are favored to promote selfishness. While the impact of patterns of dispersal on the evolution of intragenomic conflict has received recent attention, the consequences of intragenomic conflict for the evolution of dispersal remain to be explored. We suggest that if the evolution of dispersal is driven at least in part by kin selection, differential relatedness of social partners via their mothers versus their fathers may lead to an intragenomic conflict, with maternal-origin genes and paternal-origin genes favoring different rates of dispersal. As an illustration, we extend a classic model of the evolution of dispersal to explore how intragenomic conflict may arise between an individual's maternal-origin and paternal-origin genes over whether that individual should disperse in order to ease kin competition. Our analysis reveals extensive potential for intragenomic conflict over dispersal and predicts that genes underpinning dispersal phenotypes may exhibit parent-of-origin-specific expression, which may facilitate their discovery.
\end{abstract}

Keywords: genetic conflict, genomic imprinting, kin selection, migration, parent-of-origin effects.

\section{Introduction}

Intragenomic conflict may arise when social partners are more related through one parent than the other, such that genes originating from the former parent are favored to promote selflessness, while genes originating from the latter parent are favored to promote selfishness (Haig 2002; Grafen 2006). Such conflicts of interest are predicted to lead to parent-of-origin-specific gene expression-or "genomic imprinting" - whereby an allele's phenotypic effect depends

* Corresponding author; e-mail: andy.gardner@st-andrews.ac.uk.

Am. Nat. 2015. Vol. 186, pp. E000-E000. (C) 2015 by The University of Chicago. 0003-0147/2015/18603-56018\$15.00. All rights reserved.

DOI: $10.1086 / 682275$ on whether it was inherited from the individual's mother or father (Moore and Haig 1991; Haig 1997). Typically, often one gene at a diploid locus will be expressed and the other silenced, rendering the individual functionally haploid at imprinted loci (Reik and Walter 2001).

An important ecological driver of intragenomic conflict is the rate and pattern of dispersal. If individuals or gametes of one sex disperse further than those of the other prior to fertilization, this may lead social partners to be more related through one parent than the other (Haig 2000; Úbeda and Gardner 2010, 2011, 2012; Van Cleve et al. 2010; Brandvain et al. 2011; Gardner 2014; Úbeda et al. 2014). However, the impact of intragenomic conflict on the evolution of dispersal remains to be explored. Dispersal is a classic social trait, which can incur significant fitness costs for the dispersing individual and may be driven, at least in part, by an indirect fitness benefit arising from the relaxation of resource competition among the individual's kin (Hamilton and May 1977; Motro 1982a, 1982b, 1983; Gandon 1999; Otto and Day 2007, chap. 12; Clobert et al. 2012; Frank 2013). Intragenomic conflict over the propensity to disperse is expected when the individual is more related to her kin through one parent than the other.

Here, we investigate the potential for differential relatedness of social partners via their mothers and their fathers to drive an intragenomic conflict of interest between maternal-origin genes and paternal-origin genes with respect to the individual's probability of dispersing, and we employ the "loudest voice prevails" principle (Haig 1996; Úbeda and Haig 2004) to explore how such intragenomic conflict may lead to imprinting of loci underpinning dispersal phenotypes. In addition to outlining general qualitative predictions that are expected to hold over a wide range of demographies, we also provide quantitative analytical results for a simple, demographically explicit model — based on Hamilton and May's (1977) scenario of the evolution of dispersal driven by kin competition - for the purpose of illustration and concreteness. 


\section{General Analysis}

Overview

In this section, we investigate how intragenomic conflict can arise with respect to the probability of dispersal as a consequence of social partners being differentially related to each other via their mother versus their father, and we explore the consequences for parent-of-origin-specific gene expression (i.e., genomic imprinting). We first outline general qualitative predictions concerning the occurrence and direction of intragenomic conflict over dispersal that are expected to hold over a wide range of demographic scenarios. We then derive general predictions from the loudestvoice-prevails principle, relating the occurrence and direction of genomic imprinting of dispersal loci to the direction of kin selection and any parent-of-origin asymmetry in genetic relatedness.

\section{Intragenomic Conflict}

To the extent that kin selection has a role to play in the evolution of dispersal, we expect that the degree of genetic relatedness between social partners will modulate the level of dispersal that is favored by natural selection. Typically, we think of dispersal being an altruistic trait, that is, favored by natural selection because it relaxes kin competition (Hamilton and May 1977), with higher relatedness leading to a higher rate of dispersal being evolutionarily favored (Frank 2013). But in principle, higher relatedness could lead to a lower rate of dispersal being favored, for example, if beneficial kin-discriminatory behavior provides sufficient incentive for kin to remain together.

Nevertheless, irrespective of the direction of the impact of relatedness on dispersal, the possibility that the degree of relatedness has some impact on the optimal level of dispersal, coupled with the possibility that an individual may be more related to her social partners through one parent than the other, means that genes originating from one parent may have a dispersal optimum that is different from that of genes originating from the other parent. Specifically, if higher relatedness is associated with a higher dispersal optimum, then (i) if social partners are more related through their mothers than their fathers, their maternalorigin genes will favor a greater rate of dispersal than will their paternal-origin genes, and (ii) if social partners are more related through their fathers than their mothers, their paternal-origin genes will favor a greater rate of dispersal than will their maternal-origin genes. The reverse predictions obtain if higher relatedness is associated with a lower dispersal optimum (see the appendix for a more formal analysis of these general predictions). This divergence in the optima of different genes residing in the same individual constitutes intragenomic conflict over dispersal.

\section{Genomic Imprinting}

Conflict between genes with different parental origin is resolved by the evolution of parent-of-origin-specific expression (Haig 1996; Úbeda and Haig 2004). Whenever such a pattern of expression is possible, maternal-origin and paternal-origin genes in conflict are expected to engage in an antagonistic escalation of their expression strategies, with the expression of genes inherited from one parent being upregulated and the expression of genes inherited from the other parent being downregulated (Haig 1996; Úbeda and Haig 2004). The final outcome of such antagonistic coevolution is the silencing of one gene and the expression of the other at its optimal level (i.e., genomic imprinting; Haig 1996; Reik 2001; Úbeda and Haig 2004).

According to the loudest-voice-prevails principle, the gene that ends up being expressed is the one that is favored to have a greater level of expression (Haig 1996; Úbeda and Haig 2004). Here we consider a gene for which a greater level of expression results in a greater probability of dispersal (i.e., a dispersal promoter). When maternalorigin genes have a dispersal optimum that is higher than that of paternal-origin genes, we predict that the paternalorigin gene will be silenced and the maternal-origin gene will win the conflict and exhibit a level of expression that corresponds to its optimal probability of dispersal, and when paternal-origin genes have a dispersal optimum that is higher than that of maternal-origin genes, we predict that the maternal-origin gene will be silenced and the paternal-origin gene will win the conflict and exhibit a level of expression that corresponds to its optimal probability of dispersal. Considering a gene for which a greater level of expression results in a lower probability of dispersal (i.e., a dispersal inhibitor), when maternal-origin genes have a dispersal optimum that is higher than that of paternal-origin genes, we predict that the maternal-origin gene will be silenced and the paternal-origin gene will win the conflict and exhibit a level of expression that corresponds to its optimal probability of dispersal, and when paternal-origin genes have a dispersal optimum that is higher than that of maternal-origin genes, we predict that the paternal-origin gene will be silenced and the maternalorigin gene will win the conflict and exhibit a level of expression that corresponds to its optimal probability of dispersal.

\section{An Illustration}

Overview

Above, we derived general qualitative predictions concerning the occurrence and direction of intragenomic conflict over dispersal and the resulting patterns of genomic imprinting that are expected to hold over a wide range of demographic scenarios. In this section, we now provide a con- 
crete illustration of these predictions in the context of a demographically explicit model. We first introduce Hamilton and May's (1977) classic model of the evolution of dispersal, driven by kin competition for breeding opportunities, and develop this to study the evolution of dispersal in the context of a diploid hermaphrodite population in which individuals may engage in some degree of selfing. We then explore how parent-of-origin asymmetries in relatedness may arise in the context of this model and how these asymmetries may be modulated by the degree of selfing, giving rise to an intragenomic conflict between an individual's maternal-origin and paternal-origin genes over the probability of dispersing. We also provide a concrete illustration and an individual-based simulation confirmation of the loudest-voice-prevails principle for genomic imprinting of loci underpinning dispersal phenotypes.

\section{Hamilton and May's Model}

Hamilton and May (1977) developed a model of the evolution of dispersal in which an infinite population is divided up into breeding patches, with each patch containing a single breeder who produces a large number of offspring by asexual propagation and then dies. These offspring then have the opportunity to either remain on their natal patch or attempt to disperse to a different, randomly chosen patch, with some dispersers dying en route. Following dispersal, one individual is chosen at random on each patch to be a breeder, and all other individuals perish without achieving reproductive success.

The expected relative fitness of a focal juvenile may therefore be written as

$$
W=x(1-c) \frac{1}{1-c z}+(1-x) \frac{1}{1-y+(1-c) z},
$$

where the probability of dispersal is $x$ for the focal juvenile, $y$ for a juvenile that is randomly chosen from the same patch, and $z$ for a juvenile that is randomly chosen from the entire population, and $c$ is the probability that a dispersing individual dies before reaching a new patch (we assume that $0<c$ $<1)$.

We use the neighbor-modulated fitness approach to kin-selection analysis (Hamilton 1964; Taylor and Frank 1996; Frank 1997, 1998; Rousset 2004; Taylor et al. 2007) to find the convergence stable (CS; Christiansen 1991; Taylor 1996) probability of dispersal, which is given by

$$
z^{*}= \begin{cases}0 & r \leq c \\ \frac{r-c}{r-c^{2}} & r>c\end{cases}
$$

where $r$ is the kin-selection coefficient of relatedness between juveniles that are born on the same patch (see the ap- pendix for details; Frank 2013). Note that the CS probability of dispersal $z^{*}$ is a monotonically increasing function of the degree of relatedness $r$ between juvenile patch mates; higher relatedness promotes dispersal.

Owing to the assumption of asexual propagation, relatedness in Hamilton and May's (1977) model is given by $r=1$, irrespective of the rate of dispersal. This is because a breeder's offspring are clonally related to each other, irrespective of whether the breeder was herself a native of the patch or immigrated from elsewhere. But, more generally, dispersal is liable to modulate the relatedness of social partners. For example, this will be the case if there are two breeders in each patch whose offspring are related to each other only to the extent that both breeders are natives. Substituting $r=1$ into equation (2) yields Hamilton and May's (1977) result, $z^{*}=1 /(1+c)$. This result is surprising in that it shows that even in the limit of lethal dispersal, half of all individuals will disperse away from their natal patch $\left(z^{*} \rightarrow 1 / 2\right.$ as $\left.c \rightarrow 1\right)$. This illustrates the potential importance of kin competition in shaping the evolution of social behaviors.

\section{Sexual Reproduction}

We now develop Hamilton and May's (1977) model to incorporate sexual reproduction (Hamilton and May 1977; Motro 1982a, 1982b, 1983; Frank 2013). We assume that all individuals are hermaphroditic, a simplification that allows us to avoid the complexities of class structure while allowing each individual to have a mother and a father (Gardner 2014). We also assume that a fraction $s$ of the individual's eggs (or ovules) are fertilized by their own sperm (or pollen) and a fraction $1-s$ are fertilized by sperm from unrelated individuals elsewhere in the population, in line with Charlesworth and Charlesworth's (1978) classic model of selfing in plants. That is, there is no dispersal of female gametes and partial dispersal of male gametes prior to fertilization. Finally, we assume that all offspring are born into their mother's patch, and it is the rate of dispersal of these offspring that we consider to be the evolving trait.

Fitness is again given by equation (1), and-assuming genes are not privy to information about their parent of origin - the CS probability of dispersal is also given by equation (2). The consequences of moving from a model of asexual reproduction to a model of sexual reproduction are captured in the coefficient of relatedness (Frank 2013), which is now given by

$$
r=\frac{(1+s)^{2}}{4}
$$

(see the appendix for details). Substituting equation (3) into equation (2) yields the CS probability of dispersal 


$$
z^{*}=\left\{\begin{array}{ll}
0 & \frac{(1+s)^{2}}{4} \leq c \\
\frac{(1+s)^{2}-4 c}{(1+s)^{2}-4 c^{2}} & \frac{(1+s)^{2}}{4}>c
\end{array},\right.
$$

as illustrated in figure $1 A$.

Since clonal relatedness arises in the context of obligate selfing $(r=1$ when $s=1)$, we recover Hamilton and May's (1977) result $z^{*}=1 /(1+c)$ in this extreme scenario. However, the probability of dispersal that is favored by natural selection is dramatically lowered if selfing is incomplete $(s<1)$, particularly when dispersal is costly (large $c)$. Indeed, over much of the parameter space $(\sim 42 \%)$, no dispersal is favored at all $\left(z^{*}=0\right.$ when $\left.c>(1+s)^{2} / 4\right)$. This illustrates the potential for less-than-clonal relatedness to undermine group-beneficial behaviors, even in the context of relatively closely related kin groups, such as groups of half-siblings (Gardner and Grafen 2009; Gardner 2015).

\section{Intragenomic Conflict}

The results given in the previous section apply when the genes underpinning dispersal encode strategies that are not facultatively adjusted according to their parental origin. In this section, we consider such facultative behavior by determining the interests of maternal-origin versus paternalorigin genes with respect to the individual's probability of dispersal. On account of the decision to disperse being modulated by an individual's genetic relatedness to her patch mates, maternal-origin and paternal-origin genes may disagree as to the probability of dispersal when the degree of genetic relatedness is different for these two sets of genes.
A

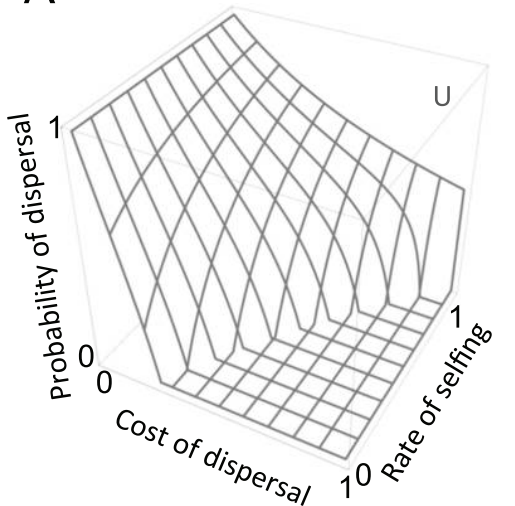

C

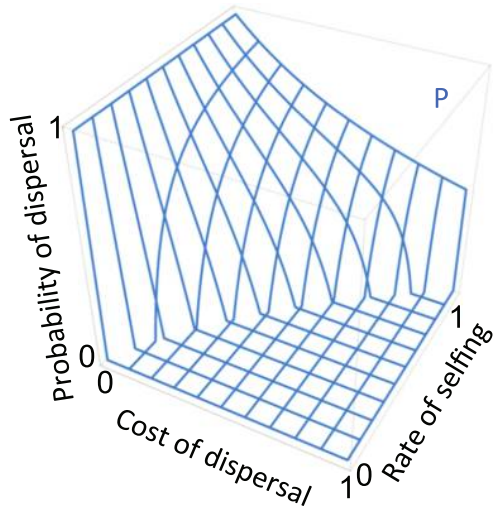

B

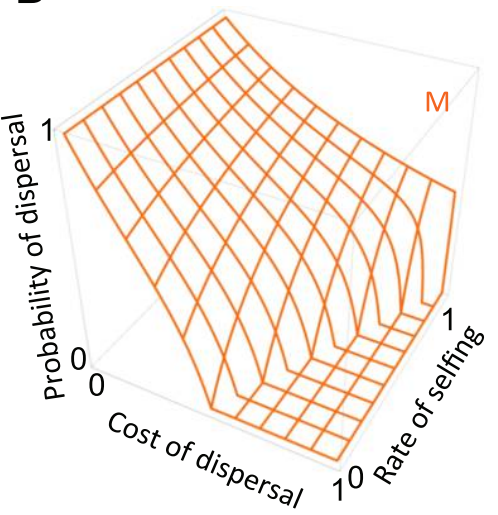

D

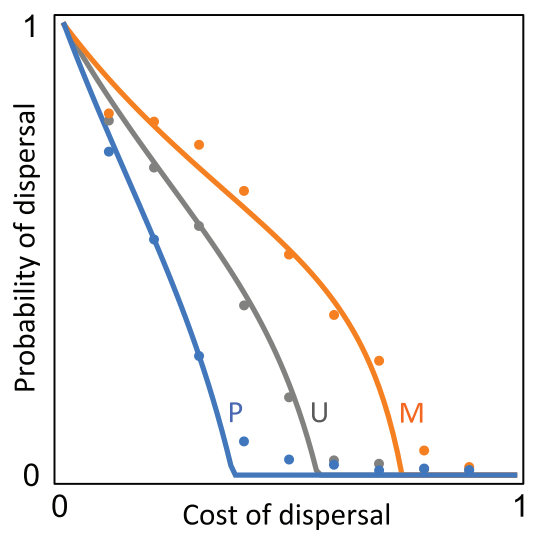

Figure 1: Intragenomic conflict over dispersal. $A$, The convergence-stable probability of dispersal for an unimprinted locus in which maternal-origin and paternal-origin genes have equal control of the dispersal phenotype as a function of the cost of dispersal and the rate of selfing. $B$, The convergence-stable probability of dispersal for an unimprinted locus in which maternal-origin genes have full control of the dispersal phenotype as a function of the cost of dispersal and the rate of selfing. $C$, The convergence-stable probability of dispersal for an unimprinted locus in which paternal-origin genes have full control of the dispersal phenotype as a function of the cost of dispersal and the rate of selfing. $D$, Analytical predictions (lines) and individual-based simulation results (filled circles) for equal control (gray, U), maternal-origin control (orange, $\mathrm{M}$ ), and paternal-origin control (blue, $\mathrm{P}$ ) for $s=0.5$. 
We find that the relatedness of an individual to her patch mates, from the perspective of her maternal-origin genes, is

$$
r_{\mathrm{M}}=\frac{1+s}{2}
$$

(see the appendix for details). Accordingly, if dispersal is controlled by maternal-origin genes, the CS probability of dispersal is found by substituting $r=r_{\mathrm{M}}$ into equation (2), which yields

$$
z_{\mathrm{M}}^{*}=\left\{\begin{array}{ll}
0 & \frac{1+s}{2} \leq c \\
\frac{1+s-2 c}{1+s-2 c^{2}} & \frac{1+s}{2}>c
\end{array},\right.
$$

as illustrated in figure $1 B$. Conversely, we find that the relatedness of an individual to her patch mates from the perspective of her paternal-origin genes is

$$
r_{\mathrm{P}}=\frac{s(1+s)}{2}
$$

(see the appendix for details). Accordingly, if dispersal is controlled by paternal-origin genes, the CS probability of dispersal is found by substituting $r=r_{\mathrm{P}}$ into equation (2), which yields

$$
z_{\mathrm{P}}^{*}=\left\{\begin{array}{ll}
0 & \frac{s(1+s)}{2} \leq c \\
\frac{s(1+s)-2 c}{s(1+s)-2 c^{2}} & \frac{s(1+s)}{2}>c
\end{array},\right.
$$

as illustrated in figure $1 C$.
Maternal-origin genes have a relatedness to patch mates that is greater than or equal to that of paternal-origin genes (i.e., $r_{\mathrm{M}} \geq r_{\mathrm{P}}$ ), and so they are favored to bring about a probability of dispersal that is greater than or equal to that of paternal-origin genes (i.e., $z_{\mathrm{M}}^{*} \geq z_{\mathrm{P}}^{*}$ ). This difference in relatedness arises because, in the context of this model, all offspring born into the same patch share the same mother, but they may have different fathers. More specifically, there is an intragenomic conflict of interest over the probability of dispersal (i.e., $z_{\mathrm{M}}^{*}>z_{\mathrm{P}}^{*}$ ) whenever the cost of dispersal is sufficiently small that dispersal is favored by at least one of these two sets of genes (i.e., $c<(1+s) / 2$ ), so long as there is incomplete selfing (i.e., $s<1$ ). Accordingly, except for the extreme scenario of obligate selfing $(s=1)$, if some nonzero probability of costly dispersal is favored by natural selection, then there is also an intragenomic conflict. This region of conflict is illustrated along with confirmatory simulation data in figure $1 D$.

\section{Genomic Imprinting}

Above, we have derived general predictions for patterns of gene expression under parent-of-origin asymmetries in relatedness. We now provide a concrete illustration of these above general predictions by applying them to our present demographically explicit scenario. In this context, we have found that maternal-origin genes have a dispersal optimum that is greater than or equal to that of paternalorigin genes. Accordingly, considering a dispersal promoter, when there is intragenomic conflict, the paternal-origin

\section{B}

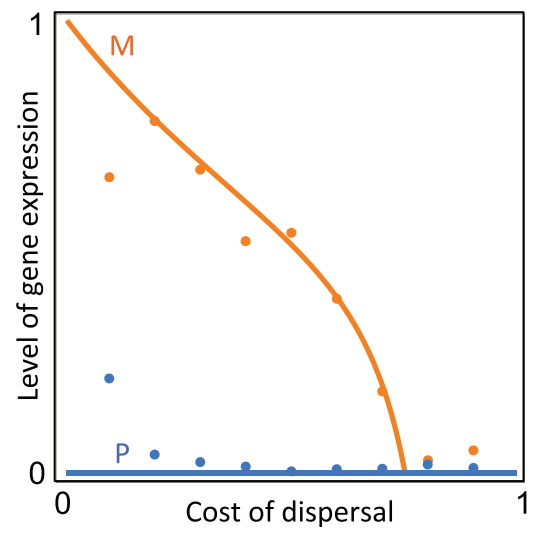

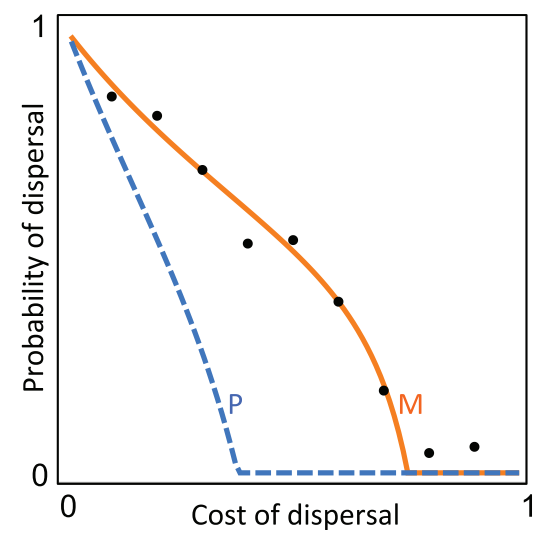

Figure 2: Genomic imprinting of a dispersal promoter. A, Analytical predictions (lines) and simulation results (filled circles) for level of gene expression for the maternal-origin (orange, $\mathrm{M}$ ) and paternal-origin (blue, $\mathrm{P}$ ) genes at a locus whose gene product promotes dispersal for $s=0.5$, where $s$ represents the rate of selfing. The individual-based simulation results provide good support for the loudest-voice-prevails principle that the paternal-origin gene is silenced and the maternal-origin gene is expressed at its optimal level when there is an intragenomic conflict of interest. $B$, The resulting probability of dispersal emerging from the individual-based simulation (filled circles) conforms to the maternal-origin gene's optimum (orange solid line, M) rather than the paternal-origin gene's optimum (blue dashed line, $\mathrm{P}$ ), indicating that the maternal-origin gene wins the conflict. 
gene is predicted to be silenced, while the maternal-origin gene is predicted to win the conflict and exhibit a level of expression that corresponds to its optimal probability of dispersal (i.e., $z_{\mathrm{M}}^{*}$; fig. 2). Considering a dispersal inhibitor, when there is intragenomic conflict, the maternal-origin gene is predicted to be silenced, while the paternal-origin gene is predicted to win the conflict and exhibit a level of expression that corresponds to its optimal probability of dispersal (i.e., $z_{\mathrm{p}}^{*}$; fig. 3).

We confirm these predictions using individual-based simulations (figs. 2, 3; see the appendix for details). The resulting simulation data are intended to check our analytical results rather than provide a formal statistical test. Nevertheless, the simulation data do show significant effects in line with our model's predictions. (i) In figure $2 A$, seven pairs of data points are presented for the level of expression of maternal-origin (orange) and paternal-origin (blue) genes within the predicted zone of intragenomic conflict, and our model predicts that-for a dispersal promoterthe maternal-origin gene should show a higher level of expression than does the paternal-origin gene, which is observed for all seven of the corresponding data points $(P=$ $\left.(1 / 2)^{7}=.0078\right)$. (ii) In figure $2 B$, seven data points are presented for the resulting probability of dispersal within the predicted zone of intragenomic conflict, and our model predicts that - for a dispersal promoter - each data point should be closer to the optimum for maternal-origin genes than that for paternal-origin genes, which is observed for all seven of the data points $\left(P=(1 / 2)^{7}=.0078\right)$. (iii) In figure $3 A$, seven pairs of data points are presented for the level of ex-

\section{A}

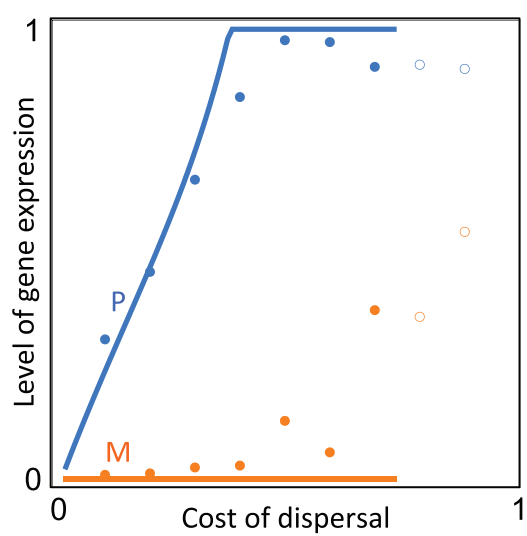

pression of maternal-origin (orange) and paternal-origin (blue) genes within the predicted zone of intragenomic conflict, and our model predicts that - for a dispersal inhibitor-the maternal-origin gene should show a lower level of expression than does the paternal-origin gene, which is observed for all seven of the corresponding data points $\left(P=(1 / 2)^{7}=.0078\right)$. (iv) In figure $3 B$, seven data points are presented for the resulting probability of dispersal within the predicted zone of intragenomic conflict, and our model predicts that - for a dispersal inhibitor - each data point should be closer to the optimum for paternal-origin genes than that for maternal-origin genes, which is observed for all seven of the data points $\left(P=(1 / 2)^{7}=.0078\right)$.

\section{Discussion}

Recent years have seen much interest in understanding how the rate and pattern of dispersal can drive the evolution of intragenomic conflict and genomic imprinting (Haig 2000; Úbeda and Gardner 2010, 2011, 2012; Van Cleve et al. 2010; Brandvain et al. 2011; Úbeda et al. 2014). Here, we have considered the reverse, specifically, the possibility for intragenomic conflict to arise with respect to the individual's decision whether to disperse. We have shown that when social partners are more related to each other through one parent than the other, an intragenomic conflict of interests may arise between an individual's maternal-origin genes and her paternal-origin genes with respect to her probability of dispersing. We have outlined general predictions relating the direction of kin selection and the asymmetry

\section{B}

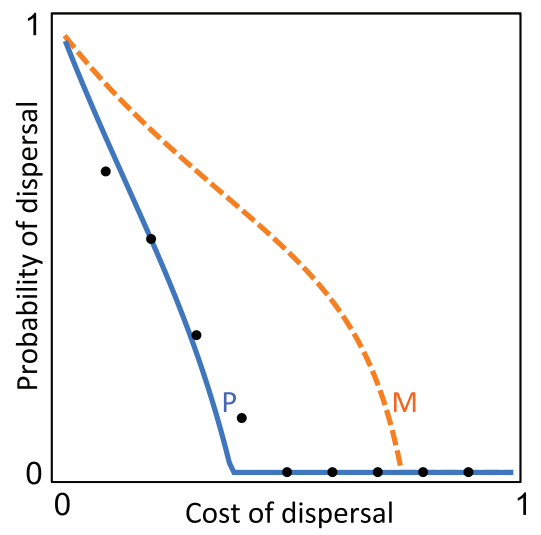

Figure 3: Genomic imprinting of a dispersal inhibiter. A, Analytical predictions (lines) and simulation results (filled circles) for level of gene expression for the maternal-origin (orange, M) and paternal-origin (blue, P) genes at a locus whose gene product inhibits dispersal, for $s=0.5$, where $s$ represents the rate of selfing. The individual-based simulation results provide good support for the loudest-voice-prevails principle that the maternal-origin gene is silenced and the paternal-origin gene is expressed at its optimal level when there is an intragenomic conflict of interest. Open circles indicate levels of gene expression outside the zone of conflict (i.e., for which the model makes no specific predictions except that the sum of the two expression levels should not be less than unity). $B$, The resulting probability of dispersal emerging from the individual-based simulation (filled circles) conforms to the paternal-origin gene's optimum (blue solid line, $\mathrm{P}$ ) rather than the maternal-origin gene's optimum (orange dashed line, M), indicating that the paternal-origin gene wins the conflict. 
in relatedness to the occurrence and direction of intragenomic conflict over dispersal and genomic imprinting of dispersal loci, and we have provided a concrete illustration of these predictions by deriving quantitative results for a simple, demographically explicit scenario in which relatedness asymmetries are modulated by selfing. We have checked these predictions using individual-based simulations and, to our knowledge, this is the first time that the logic of the loudest-voice-prevails principle of genomic imprinting (Haig 1996) has been checked in this way. These predictions, taken together with crossing experiments and gene expression data, may facilitate future discovery of genes underpinning dispersal phenotypes.

The purpose of our demographically explicit model has been to illustrate how intragenomic conflict may arise over dispersal, driving the evolution of genomic imprinting, and not to make general predictions as to the direction of imprint, as this detail will vary according to a species' ecology and demography. For instance, in this simple model, the relatedness of an individual to her patch mates with respect to her maternal-origin genes is always greater than (or equal to) that with respect to her paternal-origin genes, but if we had considered a scenario in which offspring were raised in their multiply mated father's patches, then relatedness to patch mates could be higher with respect to paternal-origin genes than with respect to maternalorigin genes, resulting in a reversal in the direction of conflict. Moreover, while our simple model explored a range of relatedness asymmetries between maternal-origin versus paternal-origin genes by altering the rate of inbreeding (in particular, the incidence of selfing), more generally, there are many demographic factors that could modulate relatedness asymmetries, including sex-specific dispersal rates, variance in mating success, and mortality schedules (Úbeda and Gardner 2010, 2011, 2012). However, our general predictions concerning the evolutionary consequences of given asymmetries in relatedness are expected to hold, irrespective of which demographic processes are causally responsible for these asymmetries.

In the context of our simple demographically explicit model, the expectation that maternal-origin genes will win the intragenomic conflict at dispersal-promoter loci while paternal-origin genes will win the intragenomic conflict at dispersal-inhibitor loci suggests that the resulting rate of dispersal may be biased toward the interests of the corresponding party if dispersal loci are predominantly promoters or predominantly inhibitors and that the interests of maternal-origin and paternal-origin genes will more or less cancel out in the aggregate if equal numbers of promoters and inhibitors underpin dispersal (Grafen 2006; Gardner and Ross 2014). Moreover, our model predicts that allelic variation segregating at such loci may have relatively large and parent-of-origin-dependent phenotypic effects.
Specifically, a knockout mutation at a dispersal-promoter locus is expected to have no impact on the phenotype when paternally derived (owing to this gene being silenced anyway) but to have a relatively large dispersal-inhibiting effect on the phenotype when maternally derived (owing to the individual failing to produce any of the dispersalpromoting gene product). Conversely, a knockout mutation at a dispersal-inhibitor locus is expected to have no phenotypic effect when maternally derived and to have a relatively large dispersal-promoting effect when paternally derived. The corresponding predictions for scenarios in which relatedness is higher with respect to the individual's paternal-origin genes are readily obtained by application of the same logic.

Currently, there appears to be no direct evidence linking imprinted genes with dispersal phenotypes, though indirect evidence is provided from two sources. First, a loss-offunction mutation in the gene Nesp55 - which is maternally expressed and paternally silenced-affects exploratory behavior in mice, with individuals that inherit the mutation from their mothers exhibiting a greater tendency to enter novel environments but a reduced tendency to remain in them (Plagge et al. 2005). Although these behaviors may modulate dispersal, the link is not entirely clear; to the extent that the wild-type gene reduces exploration, it could be interpreted as a dispersal inhibitor, but to the extent that the wild-type gene increases the likelihood of remaining in a novel environment, it could be interpreted as a dispersal promoter. Moreover, the paucity of data on the ancestral demography of these laboratory mice makes it unclear as to whether relatedness among social partners has been higher through their matrilines than through their patrilines (Úbeda and Gardner 2015). For example, owing to greater variance in male reproductive success, with a single male siring the litters of multiple females, social partners may be more related through their fathers than their mothers (Úbeda and Gardner 2010, 2011, 2012). For these reasons, it is difficult to assess whether the direction of imprint of Nesp55 is in line with theoretical predictions.

Second, male and female mice are able to discriminate between urinary odors originating from maternal versus paternal relations, showing avoidance of the former but not the latter, and this behavior has been interpreted as involving imprinted genes and having evolved in order to facilitate dispersal and outbreeding (Isles et al. 2001). Although it is difficult to bring the specific details of these empirical examples to bear on the general theory of parentof-origin intragenomic conflicts over dispersal, both of these sources of evidence do lend support to our basic assumption that genes underpinning dispersal phenotypes can exhibit imprinting and to our general prediction that genes underpinning dispersal phenotypes may evolve to exhibit parent-of-origin-specific expression. 
For reasons of simplicity, our main focus has been on Hamilton and May's (1977) demographic scenario in which there is only a single breeder in each patch. This has enabled relatively simple and explicit analytical expressions for the levels of dispersal favored by genes of different parental origin. In the context of this illustrative model, we have also assumed a diploid hermaphroditic mode of reproduction to avoid the complications of individuals belonging to different sex classes (Gardner 2014). However, there exists a very rich literature on the evolution of dispersal that has incorporated complexities such as separate sexes (Hamilton and May 1977), sex-biased dispersal (Leturque and Rousset 2004), individuals of different ages (Ronce et al. 2000; Cotto et al. 2013), and multiple (and variable numbers of) breeders in each patch (Leturque and Rousset 2002). Accordingly, the present demographically explicit model could be extended in many different directions with corresponding impact on the existence, direction, and intensity of intragenomic conflict. It is important to note that, while the existence of a mismatch between the dispersal optima of an individual's maternal-origin and paternal-origin genes underpins the prediction of genomic imprinting, and while the direction of this mismatch underpins the prediction of the direction of imprint, the loudest-voice-prevails principle predicts total silencing of the gene inherited from one parent - and optimal expression of the gene inherited from the other parent-irrespective of the intensity of the intragenomic conflict.

\section{Acknowledgments}

We thank two reviewers for helpful comments. A.G. is supported by an Independent Research Fellowship from the Natural Environment Research Council (grant NE/ K009524/1).

\section{APPENDIX}

\section{Mathematical Details}

\section{General Predictions}

In general, an individual's fitness may be written as $W(x$, $y, z)$, where $x$ denotes the probability that she will disperse, $y$ denotes the average probability of dispersal among her social partners, and $z$ denotes the average probability of dispersal among all the individuals in the population. Consider a locus $G$ that influences the probability of dispersing, and denote by $g$ the genic value of a gene drawn at random-from this locus - from the population. An increase in average genic value is favored by natural selection if $\mathrm{d} W / \mathrm{d} g>0$, where $W$ is the relative fitness of the individual carrying the gene. Following the approach of Taylor and Frank (1996; see also Taylor 1996; Frank 1997, 1998;
Rousset 2004; Taylor et al. 2007), and assuming that a gene's impact on the phenotype does not depend on its parent of origin, we may write $\mathrm{d} W / \mathrm{d} g=(\partial W / \partial x) \times(\mathrm{d} x / \mathrm{d} G) \times$ $(\mathrm{d} G / \mathrm{d} g)+(\partial W / \partial y) \times\left(\mathrm{d} y / \mathrm{d} G^{\prime}\right) \times\left(\mathrm{d} G^{\prime} / \mathrm{d} g\right)$, where $G$ is the individual's genetic breeding value for the dispersal trait (being the average of the genic values for the genes that she carries at locus $G$ ), $G^{\prime}$ is the average genetic value of the individual's social partners (i.e., the juveniles born in the same patch), and all derivatives are evaluated at $x=y=z$. Hence, $\mathrm{d} x / \mathrm{d} G=\mathrm{d} y / \mathrm{d} G^{\prime}=\gamma$ describes the mapping between genotype and phenotype, $\mathrm{d} G / \mathrm{d} g=p$ describes the consanguinity of an individual to herself, and $\mathrm{d} G^{\prime} / \mathrm{d} g=p^{\prime}$ describes the consanguinity of an individual to her social partners (Bulmer 1994). Accordingly, natural selection favors an increase in the probability of dispersal if $-C(z)+$ $B(z) r>0$, where $-C(z)=\partial W / \partial x, B(z)=\partial W / \partial y$, and $r=p^{\prime} / p$ is the kin-selection coefficient of relatedness (Bulmer 1994).

We now relax the above assumption that the gene's impact on the phenotype is independent of its parent of origin. If only the maternal-origin gene at locus $\mathrm{G}$ affects the individual's dispersal phenotype, then we must write $\mathrm{d} W / \mathrm{d} g=(\partial W / \partial x) \times\left(\mathrm{d} x / \mathrm{d} G_{\mathrm{M}}\right) \times\left(\mathrm{d} G_{\mathrm{M}} / \mathrm{d} g\right)+(\partial W / \partial y) \times$ $\left(\mathrm{d} y / \mathrm{d} G_{\mathrm{M}}^{\prime}\right) \times\left(\mathrm{d} G_{\mathrm{M}}^{\prime} / \mathrm{d} g\right)$, where $G_{\mathrm{M}}$ is the genic value of the individual's maternal-origin gene at locus $\mathrm{G}, G_{\mathrm{M}}^{\prime}$ is the average genic value of the individual's social partners' maternalorigin genes at locus $\mathrm{G}, \mathrm{d} x / \mathrm{d} G_{\mathrm{M}}=\mathrm{d} y / \mathrm{d} G_{\mathrm{M}}^{\prime}=\gamma_{\mathrm{M}}$ describes the mapping between maternal-origin gene and phenotype, $\mathrm{d} G_{\mathrm{M}} / \mathrm{d} g=p_{\mathrm{M}}=p$ is the consanguinity of the gene to the individual's maternal-origin gene, and $\mathrm{d} G_{\mathrm{M}}^{\prime} / \mathrm{d} g=p_{\mathrm{M}}^{\prime}$ is the consanguinity of the gene to the individual's social partners' maternal-origin genes. Accordingly, natural selection favors an increase in the probability of dispersal if $-C(z)+$ $B(z) r_{\mathrm{M}}>0$, where $r_{\mathrm{M}}=p_{\mathrm{M}}^{\prime} / p_{\mathrm{M}}$. Using the same logic, if only the individual's paternal-origin gene at locus $\mathrm{G}$ affects the dispersal phenotype, then the condition for increase is $-C(z)+B(z) r_{\mathrm{P}}>0$, where $r_{\mathrm{P}}$ is the coefficient of relatedness for paternal-origin genes.

The condition for natural selection to favor an increase in the probability of dispersal is exactly the same, irrespective of whether dispersal is controlled by maternalorigin or paternal-origin genes or whether control is equally shared between both of these parties, except that the coefficient of relatedness differs in each case. Formally, the condition for increase is $-C(z)+B(z) \rho>0$, where $\rho=r_{\mathrm{M}}$ for maternal-origin genetic control, $\rho=r_{\mathrm{P}}$ for paternal-origin genetic control, and $\rho=r$ for equal control. Defining a function $J\left(\zeta^{*}, \rho\right)=-C\left(\zeta^{*}\right)+B\left(\zeta^{*}\right) \rho$, where $\zeta^{*}$ represents a dispersal optimum (more formally, a convergence stable strategy; Christiansen 1991; Taylor 1996), we have $J\left(\zeta^{*}\right.$, $\rho)=0$ and $\partial J / \partial \zeta^{*}<0$. Consequently, we may write $\mathrm{d} J /$ $\mathrm{d} \rho=(\partial J / \partial \rho)+\left(\partial J / \partial \zeta^{*}\right) \times\left(\mathrm{d} \zeta^{*} / \mathrm{d} \rho\right)=0$, which rearranges to $\mathrm{d} \zeta^{*} / \mathrm{d} \rho=-(\partial J / \partial \rho) /\left(\partial J / \partial \zeta^{*}\right)$, and hence we obtain 
$S\left(\mathrm{~d} \zeta^{*} / \mathrm{d} \rho\right)=S(\partial J / \partial \rho)=S\left(B\left(\zeta^{*}\right)\right)$, where the function $S$ returns the sign (i.e., positive or negative or zero) of its argument (cf. Pen 2000). Accordingly, if the dispersal of social partners improves the individual's fitness $(B>0)$, then higher relatedness is associated with a higher dispersal optimum $\left(\mathrm{d} \zeta^{*} / \mathrm{d} \rho>0\right)$; if the dispersal of social partners decreases the individual's fitness $(B<0)$, then higher relatedness is associated with a lower dispersal optimum $\left(\mathrm{d} \zeta^{*} / \mathrm{d} \rho<0\right)$; and if the dispersal of social partners does not affect the individual's fitness $(B=0)$, then higher relatedness is not associated with a higher or lower dispersal opti$\operatorname{mum}\left(\mathrm{d} \zeta^{*} / \mathrm{d} \rho=0\right)$.

In other words, (i) if the dispersal of social partners improves an individual's fitness $(B>0)$, then the dispersal optimum is higher for maternal-origin genes than it is for paternal-origin genes $\left(z_{\mathrm{M}}^{*}>z_{\mathrm{P}}^{*}\right)$ when relatedness is higher for the former than for the latter $\left(r_{\mathrm{M}}>r_{\mathrm{P}}\right)$, and the dispersal optimum is lower for maternal-origin genes than it is for paternal-origin genes $\left(z_{\mathrm{M}}^{*}<z_{\mathrm{P}}^{*}\right)$ when relatedness is lower for the former than for the latter $\left(r_{\mathrm{M}}>r_{\mathrm{P}}\right)$; (ii) if the dispersal of social partners decreases the individual's fitness $(B<0)$, then the dispersal optimum is lower for maternalorigin genes than it is for paternal-origin genes $\left(z_{\mathrm{M}}^{*}<z_{\mathrm{P}}^{*}\right)$ when relatedness is higher for the former than for the latter $\left(r_{\mathrm{M}}>r_{\mathrm{P}}\right)$, and the dispersal optimum is higher for maternalorigin genes than it is for paternal-origin genes $\left(z_{\mathrm{M}}^{*}>z_{\mathrm{P}}^{*}\right)$ when relatedness is lower for the former than for the latter $\left(r_{\mathrm{M}}>r_{\mathrm{P}}\right)$; and (iii) if the dispersal of social partners does not affect the individual's fitness $(B=0)$, then the dispersal optimum for maternal-origin genes is equal to that for paternal-origin genes $\left(z_{\mathrm{M}}^{*}=z_{\mathrm{P}}^{*}\right)$, irrespective of any differences in relatedness.

\section{Hamilton and May's Model}

From equation (1) of the main text, we have $\partial W / \partial x=$ $-c /(1-c z)$ and $\partial W / \partial y=(1-z) /(1-c z)^{2}$, and hence natural selection favors an increase in the probability of dispersal if $-c /(1-c z)+(1-z) /(1-c z)^{2} r>0$. This condition is satisfied at $z=0$ when $r>c$ and is never satisfied at $z=1$. Converting the condition for increase into an equation and rearranging yields $z=(r-c) /\left(r-c^{2}\right)$. Accordingly, the convergence stable level of dispersal is $z^{*}=$ 0 when $r \leq c$ and $z^{*}=(r-c) /\left(r-c^{2}\right)$ when $r>c$, as given in equation (2) of the main text (more formally, a secondderivative condition for convergence stability is given by Taylor 1996).

\section{Relatedness}

For the purpose of calculating consanguinity and relatedness, we assume that all consanguinity coefficients have attained their (quasi-)equilibrium values. The consanguin- ity of an individual to herself $p$ is defined by drawing two genes at random from her from the same locus, with replacement, and is equal to the probability that these two genes are identical by descent (Bulmer 1994). For a diploid individual in a sexual population, with probability one-half the same gene-either the maternal-origin or the paternal-origin gene-is drawn twice, meaning that the genes are identical by descent with probability 1 , and with probability one-half the maternal-origin and the paternalorigin gene are both drawn, meaning that the genes are identical by descent with probability $f$, where $f$ is the consanguinity of mating partners (inbreeding coefficient). Hence, consanguinity to self is $p=(1+f) / 2$. Since an individual mates with herself with probability $s$ and mates with a nonrelative with probability $1-s$, the consanguinity of mating partners is $f=s p$. Accordingly, $p=(1+s p) / 2$ or, equivalently, $p=1 /(2-s)$. Similarly, the consanguinity of social partners $p^{\prime}$ is defined by drawing a gene at random from each of the two individuals from the same locus and is equal to the probability that these genes are identical by descent (Bulmer 1994). For a diploid individual in a sexual population, with probability one-fourth the maternal-origin genes are drawn from both individuals, and since they share the same mother, the probability of identity by descent is $p$; with probability one-half the maternal-origin gene is drawn from one individual and the paternal-origin gene is drawn from the other, and since they share the same mother, the probability of identity by descent is $s p$; and with probability one-fourth the paternal-origin genes are drawn from both individuals, and since they share the same mother, the probability of identity by descent is $s^{2} p$. Accordingly, $p^{\prime}=(1 / 4)$ $p+(1 / 2) s p+(1 / 4) s^{2} p$ or, equivalently, $p^{\prime}=(1 / 4)(1+s)^{2} p$. Finally, relatedness describes the consanguinity of social partners, scaled relative to the consanguinity to self: $r=p^{\prime} / p$ or, in terms of model parameters, $r=(1+s)^{2} / 4$, as given in equation (3) of the main text. Relatedness from the perspective of an individual's maternal-origin genes is given by $r_{\mathrm{M}}=p_{M}^{\prime} / p$, where $p_{\mathrm{M}}^{\prime}$ is the consanguinity of social partners conditional on drawing the maternal-origin gene from the first individual. In other words, $p_{M}^{\prime}=(1 / 2) p+(1 / 2) s p$, and so $r_{\mathrm{M}}=(1+s) / 2$, as given in equation (5) of the main text. Relatedness from the perspective of an individual's paternal-origin genes is given by $r_{\mathrm{P}}=p_{\mathrm{P}}^{\prime} / p$, where $p_{\mathrm{P}}^{\prime}$ is the consanguinity of social partners conditional on drawing the paternal-origin gene from the first individual. In other words, $p_{\mathrm{P}}^{\prime}=(1 / 2) s p+(1 / 2) s^{2} p$, and so $r_{\mathrm{P}}=(s(1+s)) / 2$, as given in equation (7) of the main text.

\section{Individual-Based Simulation}

We consider a population of 1,000 patches in which each patch contains a single breeder and each breeder produces 100 offspring, with one randomly chosen offspring being 
constrained to remain in the natal patch (to ensure at leas one individual per patch after dispersal) and each of the other offspring dispersing with probabilities determined by their genotypes. In the first set of simulation models, alleles are represented by a real-valued number $\nu$ between 0 and 1 , and the individual's probability of dispersing is given by either the allelic value of their maternal-origin gene $\nu_{\mathrm{M}}$ (maternal-origin gene controls dispersal), the allelic value of their paternal-origin gene $\nu_{\mathrm{P}}$ (paternal-origin gene ues $\left(\nu_{\mathrm{M}}+\nu_{\mathrm{P}}\right) / 2$ (both genes control dispersal). In the sec ond set of simulation models, alleles are represented by two real-valued numbers $\mu$ and $\pi$ between 0 and 1 pertain ing to level of expression when maternal origin versus paternal origin, respectively, and the individual's probability of dispersing is given by either the sum of its two expression levels $\mu_{\mathrm{M}}+\pi_{\mathrm{P}}$ (or 1 , whichever is smaller; dispersa ${ }^{1}$ promoter) or the complement of the sum of its two expression levels $1-\left(\mu_{\mathrm{M}}+\pi_{\mathrm{P}}\right)$ (or 0 , whichever is larger; dispersal inhibitor). At initialization, every $\nu, \mu$, and $\pi$ valur is assigned a random uniformly distributed real value between 0 and 1 . For the first set of simulation models, dur ing the production of each offspring, each allele mutates to a random, uniformly distributed real value between $b$ and 1 with probability .001 . For the second set of simulation models, each parent-specific level of expression of eack allele mutates to a random, uniformly distributed real value between 0 and 1 with probability .001 . We track 1,000 generations of evolution, and average allelic values, expression levels, and probabilities of dispersal are calculated in the final generation. The simulation data points given in figures 1,2 , and 3 each derive from a single simulation replicate. Simulation code is provided in a zip file, availabl online. $^{1}$ controls dispersal), or the average of their two allelic val-

Cotto, O., I. Olivieri, and O. Ronce. 2013. Optimal life-history schedule in a metapopulation with juvenile dispersal. Journal of Evolutionary Biology 26:944-954.

Frank, S. A. 1997. Multivariate analysis of correlated selection and kin selection, with an ESS maximization method. Journal of Theoretical Biology 189:307-316.

. 1998. Foundations of social evolution. Princeton University Press, Princeton, NJ.

. 2013. Natural selection. VII. History and interpretation of kin selection theory. Journal of Evolutionary Biology 26:1151-1184.

Gandon, S. 1999. Kin competition, the cost of inbreeding and the evolution of dispersal. Journal of Theoretical Biology 200:345-364.

$\rightarrow$ Gardner, A. 2014. Genomic imprinting and the units of adaptation. Heredity 113:104-111.

. 2015. The genetical theory of multilevel selection. Journal of Evolutionary Biology 28:305-319.

Gardner, A., and A. Grafen. 2009. Capturing the superorganism: a formal theory of group adaptation. Journal of Evolutionary Biology 22:659-671.

Gardner, A., and L. Ross. 2014. Mating ecology explains patterns of genome elimination. Ecology Letters 17:1602-1612. doi:10.1111 /ele.12383.

Grafen, A. 2006. Optimization of inclusive fitness. Journal of Theoretical Biology 238:541-563.

$\rightarrow$ Haig, D. 1996. Placental hormones, genomic imprinting, and maternalfetal communication. Journal of Evolutionary Biology 9:357-380.

$\rightarrow$ 1997. Parental antagonism, relatedness asymmetries, and genomic imprinting. Proceedings of the Royal Society B: Biological Sciences 264:1657-1662.

2000. Genomic imprinting, sex-biased dispersal, and social behavior. In D. LeCroy and P. Moller, eds. Evolutionary perspectives on human reproductive behavior, special issue, Annals of the New York Academy of Sciences 907:149-163.

2002. Genomic imprinting and kinship. Rutgers University Press, New Brunswick, NJ.

$\rightarrow$ Hamilton, W. D. 1964. The genetical evolution of social behaviour. I, II. Journal of Theoretical Biology 7:1-52.

$\rightarrow$ Hamilton, W. D., and R. M. May. 1977. Dispersal in stable habitats. Nature 269:578-581.

$\rightarrow$ Isles, A. R., M. J. Baum, D. Ma, E. B. Keverne, and N. D. Allen. 2001. Genetic imprinting: urinary odour preferences in mice. Nature 409: 783-784.

\section{Literature Cited}

Brandvain, Y., J. Van Cleve, F. Úbeda, and J. Wilkins. 2011. Demography, kinship, and the evolving theory of genomic imprinting. Trends in Genetics 27:251-269.

Bulmer, M. G. 1994. Theoretical evolutionary ecology. Sinauer, Sunderland, MA.

$\rightarrow$ Charlesworth, B., and D. Charlesworth. 1978. A model for the evolution of dioecy and gynodioecy. American Naturalist 112:975997.

$\rightarrow$ Christiansen, F. B. 1991. On conditions for evolutionary stability fo a continuously varying character. American Naturalist 138:37-50 Clobert, J., M. Baguette, T. G. Benton, and J. M. Bullock. 2012. Dispersal ecology and evolution. Oxford University Press, Oxford.

1. Code that appears in The American Naturalist is provided as a convenience to the readers. It has not necessarily been tested as part of the peer re view. $\rightarrow$ Leturque, H., and F. Rousset. 2002. Dispersal, kin competition, and the ideal free distribution in a spatially heterogeneous population. Theoretical Population Biology 62:169-180.

$\rightarrow \quad$ 2004. Intersexual competition as an explanation for sex-ratio and dispersal biases in polygynous species. Evolution 58:2398-2408.

$\rightarrow$ Moore, T., and D. Haig. 1991. Genomic imprinting in mammalian development: a parental tug-of-war. Trends in Genetics 7:45-49.

$\rightarrow$ Motro, U. 1982a. Optimal rates of dispersal. I. Haploid populations. Theoretical Population Biology 21:394-411.

$\rightarrow \quad$. 1982b. Optimal rates of dispersal. II. Diploid populations. Theoretical Population Biology 21:412-429.

$\rightarrow \quad$. 1983. Optimal rates of dispersal. III. Parent-offspring conflict. Theoretical Population Biology 23:159-168.

Otto, S. P., and T. Day. 2007. A biologist's guide to mathematical modeling in ecology and evolution. Princeton University Press, Princeton, NJ.

$\rightarrow$ Pen, I. 2000. Reproductive effort in viscous populations. Evolution 54:293-297. 
$\rightarrow$ Plagge, A., A. R. Isles, E. Gordon, T. Humby, W. Dean, S. Gritsch $\rightarrow$ R. Fischer-Colbrie, L. S. Wilkinson, and G. Kelsey. 2005. Imprinted Nesp55 influences behavioural reactivity to novel environments Molecular and Cellular Biology 25:3019-3026.

$\rightarrow$ Reik, W., and J. Walter. 2001. Genomic imprinting: parental influ ence on the genome. Nature Reviews Genetics 2:21-32.

$\rightarrow$ Ronce, O., S. Gandon, and F. Rousset. 2000. Kin selection and nata dispersal in an age-structured population. Theoretical Population Biology 58:143-159.

Rousset, F. 2004. Genetic structure and selection in subdivided populations. Princeton University Press, Princeton, NJ.

$\rightarrow$ Taylor, P. D. 1996. Inclusive fitness arguments in genetic models of behaviour. Journal of Mathematical Biology 34:654-674.

$\rightarrow$ Taylor, P. D., and S. A. Frank. 1996. How to make a kin selection model. Journal of Theoretical Biology 180:27-37.

$\rightarrow$ Taylor, P. D., G. Wild, and A. Gardner. 2007. Direct fitness or inclusive fitness: how shall we model kin selection? Journal of Evolutionary Biology 20:301-309.
Úbeda, F., and A. Gardner. 2010. A model for genomic imprinting in the social brain: juveniles. Evolution 64:2587-2600.

2011. A model for genomic imprinting in the social brain: adults. Evolution 65:462-475.

- 2012. A model for genomic imprinting in the social brain: elders. Evolution 66:1567-1581.

. 2015. Mother and offspring in conflict: why not? PLoS Biology 13:e1002084

$\rightarrow$ Úbeda, F., and D. Haig. 2004. Dividing the child. Genetica 117:103110.

$\rightarrow$ Úbeda, F., H. Ohtsuki, and A. Gardner. 2014. Ecology drives intragenomic conflict over menopause. Ecology Letters 17:165-174.

$\rightarrow$ Van Cleve, J., M. W. Feldman, and L. Lehmann. 2010. How demography, life history, and kinship shape the evolution of genomic imprinting. American Naturalist 176:440-455.

Associate Editor: Yannis Michalakis Editor: Judith L. Bronstein 